tender joint count (SJC and TJC, respectively), and acute phase reactants (ESR and CRP) were observed between baseline and 8 weeks (table 1). By IHC, TCZ induced significant decrease in the number of CD3, C03 and CD55, but not in the number of the other CP, PAD2, PAD4 and CD68 (table 1). No significant correlations between reduction in disease activity (by $\triangle \mathrm{DAS} 28, \triangle \mathrm{SJC}, \triangle \mathrm{TJC}, \triangle \mathrm{ESR}, \Delta \mathrm{CRP}$ ) and reduction in CP, CD68 or CD3 were found.

Conclusions Treatment with IL6R blockade reduced the number of synovial tissue $\mathrm{T}$ cells. This was observed in the responders but not in non-responders. Significant reduction in citrullination as assessed by expression of 1325:C03 but not 1325:B09. No significant reduction in macrophages was observed.

Disclosure of interest None declared

\section{P063 EFFECT OF ADIPOKINES AND IL-17 ON SYNOVIAL FIBROBLAST FROM DIFFERENT RHEUMATIC DISEASE BACKGROUNDS}

${ }^{1} \mathrm{KW}$ Frommer*, ${ }^{2} \mathrm{~S}$ Rehart, ${ }^{3} \mathrm{M}$ Sauerbier, ${ }^{1} \mathrm{U}$ Müller-Ladner, ${ }^{4} \mathrm{E}$ Neumann. ${ }^{1} D e p t$. of Rheumatology and Clinical Immunology, Campus Kerckhoff, Justus-Liebig-University Giessen, Bad Nauheim; ' ${ }^{2}$ Dept. of Orthopaedics and Trauma Surgery, Agaplesion Markus Hospital; ${ }^{3}$ Dept. of Plastic, Hand and Reconstructive Surgery, BGU Frankfurt, Frankfurt; ${ }^{4}$ Dept. of Orthopaedics and Orthopaedic Surgery, Campus Kerckhoff, Justus-Liebig-University Giessen, Bad Nauheim, Germany

\subsection{6/annrheumdis-2018-EWRR2018.82}

Introduction Rheumatoid arthritis (RA) and psoriatic arthritis (PsA) have several features in common but also possess distinct differences. Synovial fibroblasts (SF) are known key effector cells in the pathophysiology of RA. We hypothesised that differential responses of SF from patients with PsA and RA to various stimuli including adipokines and cytokines may contribute to those differences. For example, IL-17 (also found in synovial tissue) is of particular therapeutic significance in PsA but not as effective in RA. Thus far, IL-17 in its isoform IL$17 \mathrm{~A}$ has been the major therapeutic target in PsA but IL-17F also plays a role in the IL-23/IL-17 axis of inflammatory diseases.

Objectives Therefore, we analysed the responses of SF from patients with PsA, RA or no rheumatic disease to IL-17A/F \pm TNF- $\alpha$ and selected adipokines.

Methods SF were isolated from patients with PsA, RA or nonrheumatic disease controls $(\mathrm{N})$, each undergoing joint surgery. PsASF, RASF and NSF were stimulated with recombinant IL$17 \mathrm{~A} / \mathrm{F}$, TNF-, visfatin, and resistin. A neutralising anti-IL-17A antibody was used to verify specificity of the IL-17A effects. Secretion of the proinflammatory cytokine IL-6 was used as the initial readout parameter and was quantified using a commerical immunoassay.

Results Stimulation with visfatin caused a strong increase in IL-6 secretion in all SF types ( $\mathrm{n}=3$ each), while resistin had no effect. Differences in responses were not statistically significant between the SF types studied. IL-17A at concentrations found in serum or synovial fluid did not induce IL-6 secretion in any of the SF. Dose-response curve analysis showed that considerably higher concentrations of IL-17A, which may occur locally in tissue, are required for the induction of IL-6 secretion. An anti-IL-17A antibody abolished the effect, thus showing that the effect is specific for IL-17A. The effects of IL-17A and IL-17F on IL-6 secretion by PsASF could be strongly amplified by a co-stimulation with TNF- $\alpha$ (IL-17A: 5-fold vs 113-fold; IL-17F: 1.7-fold vs 39-fold; TNF- $\alpha$ alone: 12 -fold). The effects were stronger for IL-17A than for IL$17 \mathrm{~F}$ with or without TNF co-stimulation. No effect of IL$17 \mathrm{~F}$ alone was observed on NSF $(n=1)$.

Conclusions SF from RA and PsA were not differentially affected by the adipokines visfatin and resistin or IL-17A when used at serum or synovial fluid concentrations. The property of IL-17F not affecting NSF but PsASF (and RASF) may be beneficial in its use as therapeutic target.

Acknowledgements This work was supported by an unrestricted educational grant from Celgene $\mathrm{GmbH}$.

Disclosure of interest None declared

\section{P064 ADIPOSE TISSUES OBTAINED FROM RA AND OA PATIENTS DIFFER IN CYTOKINE AND CHEMOKINE SECRETION}

M Plebanczyk*, A Radzikowska, T Burakowski, W Maslinski, E Kontny. National Institute of Geriatrics, Rheumatology and Rehabilitation, Warsaw, Poland

\subsection{6/annrheumdis-2018-EWRR2018.83}

Introduction Rheumatoid arthritis (RA) and osteoarthritis (OA) lead to joint destruction and disability. Although both diseases are characterised by inflammation of the joints as well as systemic inflammation, their pathogenesis is different. Despite the progress in the treatment both diseases are still incurable. Articular adipose tissue (AAT) and subcutaneous adipose tissue (ScAT) are supposed to contribute to joint and systemic inflammation, respectively. Our previous work showed that AAT from RA patients synthesises factors relevant to disease pathogenesis and/or progression and this secretion is usually higher from articular than subcutaneous adipose tissue. ${ }^{1,2}$

Objectives The aims of present work were (i) to investigate whether AAT obtained from OA patients is also more active than ScAT and (ii) to compare secretory activity of adipose tissues from RA and OA patients.

Methods AAT and ScAT explants obtained from OA [ $\mathrm{n}=44$; female (F)/male $(\mathrm{M})=36 / 8$; age $=62$ (mean) (35-71) (min$\max )$ ] and RA ( $\mathrm{n}=43 ; \mathrm{F} / \mathrm{M}=35 / 8 ;$ age $=54$ (31-70)] patients during knee joint replacement surgery were cultured $(100 \mathrm{mg} /$ $\mathrm{ml}$ ) for 24 hours in DMEM. Concentrations of proinflammatory (IL-6, TNF), anti-inflammatory (IL-1Ra, IL-10, TGF $\beta$ ) cytokines, chemokines (CCL2, CCL5) and metalloproteinase MMP-3 was measured in culture supernatants by ELISA.

Results In both diseases AAT secreted spontaneously more IL1Ra, TGF $\beta$ and MMP-3 than ScAT while the release of other factors was minute (TNF, IL-10, CCL5) or moderate (CCL2) and did not differ between tissues. The only exception was IL-6 produced in larger quantity by AAT than ScAT from OA patients. Both adipose tissues from OA patients released significantly more TNF, IL-1Ra and CCL2 than tissues from RA patients. Moreover, AAT from OA produced more MMP-3 than respective tissue of RA patients while rheumatoid ScAT secreted more TGF $\beta$. We did not find differences in basal secretion of IL-6, IL-10 and CCL5 between diseases.

Conclusions Despite the fact, that similarly to RA the secretion of cytokines and chemokines in OA was usually higher in AAT than in ScAT, also the latter tissue released considerable quantity of tested factors and thus may contribute to systemic inflammation. Unexpectedly, our results give also evidence that basal secretory activity of adipose tissues from OA patients is usually higher than from RA patients. It is possibly caused by 
different anti-inflammatory treatment, stronger in RA than OA patients, with resulting poor control of inflammation in OA.

\section{REFERENCES}

1. Kontny $\mathrm{E}$, et al. Ann Rheum Dis 2012;71(2):262-267.

2. Kontny $E$, et al. Rheumatology 2013;52:2158-2167.

Acknowledgements This work was funded by the National Science Centre (grant number 2012/N/NZ5/00074).

Disclosure of interest None declared

\section{P065 INSULIN-LIKE GROWTH FACTOR 1 RECEPTOR REGULATES THE PHENOTYPE AND FUNCTION OF CD21+ B CELLS}

M Erlandsson*, C Wasen, G Gravina, MI Bokarewa. Rheumatology and Inflammation Research, Gothenburg University, Gothenburg, Sweden

\subsection{6/annrheumdis-2018-EWRR2018.84}

Introduction Ligand to the inducible $\mathrm{T}$ cell costimulator (ICOSL) on B cells is essential for the ICOS-dependent follicular recruitment of activated $\mathrm{T}$ cells. In patients with Rheumatoid arthritis (RA) the IGF1-IGF1R axis is altered. Inhibition of IGF1R alleviated arthritis by reducing IL-6-dependent formation of Th17 cells. Here we study the role of IGF1R on CD21 + cells in experimental arthritis.

Methods Female Balb/c mice were immunised with methylated BSA or with CII. Consequences of the IGF-1R inhibition for arthritis were studied in mBSA and CII-immunised mice treated with NT157 compound promoting degradation of insulin receptor substrates (mBSA) or using shRNA producing construct (mBSA + CII). At termination three sub-populations of CD21 + cells were analysed: follicular dendritic cells (FDc, CD21 +CD19-CXCR5-); marginal zone B cells (MZBc, CD21+CD19+CXCR5+). Supernatants of LPS-stimulated splenocytes and serum were analysed for production of antigen specific and autoantibodies.

Results In spleen of mBSA-immunised mice, ICOSL expression on CD21 + cells correlated to IGF1R $(r=0.70)$. Inhibition of IGF1R induced a $20 \%$ reduction in ICOSL expression in all CD21 + subsets followed by an increase in MZBc $(p=0.003)$, while $\mathrm{FDc}$ and $\mathrm{FBc}$ were unchanged. ICOSL +MZBc were mostly IgMhi, while ICOSL $+\mathrm{FBc}$ were mostly IgDhi. Inhibition of IGF1R had no effect on the expression of ICOS +on CD4 $\mathrm{T}$ cells and the subset of CXCR5 +follicular T cells. Reduction of the ICOSL +CD21 $+\mathrm{B}$ cells, reduced production of mBSA-specific IgM and increased production of autoreactive RF-IgM levels.

Conclusions The study shows that IGF1R controls expression of ICOSL on CD21 + cells. ICOSL on MZBc is required to balance between antigen-specific response and autoantibody production.

Disclosure of interest None declared CD21 +CD19+CXCR5-); follicular B cells (FBc,

\section{P066 ABSTRACT WITHDRAWN}

M Noack*, P Miossec. Immunogenomic and Inflammation Unit, Hospital Edouard Herriot, University Claude Bernard Lyon 1, Lyon, France

\subsection{6/annrheumdis-2018-EWRR2018.85}

Introduction IL-17, mainly produced by Th17 cells, is a major pro-inflammatory cytokine involved in several chronic inflammatory diseases. Furthermore, during chronic inflammation, immune cells, notably Th17 cells, migrate to the inflammatory site, synovium or skin for example, and interact with the local mesenchymal cells (synoviocytes or skin fibroblasts).

Objectives The aim is to study and compare the effect of cellular interactions between immune cells and mesenchymal cells (MC) from different origins on pro-inflammatory cytokine production, with a focus on IL-17, and to identify the involved mechanisms.

Methods A co-culture system with MC (synoviocytes or skin fibroblasts) and PBMC was developed. MC were cultured overnight and PBMC were seeded at a 5:1 ratio for 48 hour, in the presence or not of TCR activation with PHA. Transwell system was used to study cell-cell contact. Monocytes were removed to study their involvement. An antibody against podoplanin was preincubated with PBMC before co-culture. Supernatants were collected at 48 hour and IL-6, IL-1 $\beta$ and IL-17 production measured by ELISA. Extracellular (CD3, CD4) and intracellular (IL-17) staining of PBMC was analysed by flow cytometry.

Results In control conditions, IL- 6 and IL- $1 \beta$ production was increased more than 20 fold and 10 fold respectively, in PBMC$\mathrm{MC}$ co-culture compared to PBMC alone $(\mathrm{p} \leq 0.05)$. No additional effect was observed with PBMC activation. Flow cytometry showed no significant difference in the percentage of Th17 cells in activated-PBMC alone or co-cultured with MC $(p=0.4)$. Conversely, IL-17 production was highly increased at least 10 fold only in co-cultures with activated-PBMC $(\mathrm{p} \leq 0.02)$. Transwell experiments confirm that cell-cell contact was critical for IL-17 secretion. The removal of monocytes highly decreased the IL-1 $\beta$ production by $80 \%-90 \%(\mathrm{p} \leq 0.05)$ with both MC, while the IL-17 secretion was decreased with skin fibroblasts by $60 \%$ but not with synoviocytes. The inhibition of podoplanin, interaction molecule involved in the modulation of IL-8 secretion during synoviocyteplatelet interactions, was tested. The addition of an anti-podoplanin antibody decreased significantly IL-17 secretion by $60 \%$, similarly with skin fibroblasts and synoviocytes.

Conclusions Cellular interactions between mesenchymal cells and immune cells play a major role in the pro-inflammatory cytokine production, leading to a heightened IL-17 secretion. The podoplanin molecule seems play a crucial role in this mechanism. Nevertheless, the inhibition of IL-17 remains only 